\title{
Estimating the Doppler centroid of SAR data
}

\section{Madsen, Søren Nørvang}

\section{Published in:}

I E E E Transactions on Aerospace and Electronic Systems

Link to article, DOI:

10.1109/7.18675

Publication date:

1989

Document Version

Publisher's PDF, also known as Version of record

Link back to DTU Orbit

Citation (APA):

Madsen, S. N. (1989). Estimating the Doppler centroid of SAR data. I E E E Transactions on Aerospace and Electronic Systems, 25(2), 134-140. https://doi.org/10.1109/7.18675

\section{General rights}

Copyright and moral rights for the publications made accessible in the public portal are retained by the authors and/or other copyright owners and it is a condition of accessing publications that users recognise and abide by the legal requirements associated with these rights.

- Users may download and print one copy of any publication from the public portal for the purpose of private study or research.

- You may not further distribute the material or use it for any profit-making activity or commercial gain

- You may freely distribute the URL identifying the publication in the public portal

If you believe that this document breaches copyright please contact us providing details, and we will remove access to the work immediately and investigate your claim 


\section{INTRODUCTION}

\section{Estimating The Doppler Centroid of SAR Data}

S. NØRVANG MADSEN

Technical University of Denmark

One of the parameters required to perform the azimuth processing of a synthetic aperture radar (SAR) image is the Doppler-shift (centroid) of the return echo signal. The Doppler centroid can be estimated from measurements of sensor trajectory and attitude or by analyzing characteristics of the received data. The latter will generally give the highest accuracy. Previously, the Doppler estination algorithm has been based on frequency-domain techniques, but this paper describes a time-domain method and highlights its advantages. In particular, a nonlinear time-domain algorithm called the "Sign-Doppler Estimator" has attractive properties.

Manuscript received September 28, 1987; revised June 8, 1988.

IEEE Log No. 26872.

Author's address: Technical University of Denmark, Electromagnetic Institute, Bldg. 348, DK 2800, Lyngby, Denmark.

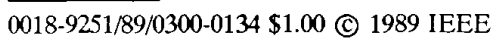

A strip map synthetic aperture radar (SAR) system is a coherent radar which can be flown either on an aircraft or a satellite. The SAR is able to produce a high-resolution radar reflectivity map of a swath alongside the platform ground track (see Fig. 1).

Strip map SAR systems are currently used for a large number of applications, ranging from Earth resource applications such as agriculture, ice mapping, pollution mapping, and geology, to military reconnaissance tasks, [1].

The two dimensions of the image are resolved as follows. Range or across-track resolution is achieved through accurate time-delay measurements using either extremely short pulses or time-dispersed phase-coded pulses. The azimuth or along-track resolution is obtained by recording the Doppler history of the individual target as it traverses the antenna beam. Basic SAR theory is described in more detail in $[1,2]$. By using the Doppler history of the targets the along-track resolution is determined by the Doppler bandwidth, offering typically an improvement of orders of magnitude compared with the resolution given by the antenna beamwidth. Hence, SAR image formation includes range pulse compression and azimuth compression.

An important parameter in relation to the azimuth SAR processing is the Doppler centroid, which is the Doppler-shift of a target positioned in the antenna boresight direction. For an airborne radar where the antenna is pointed perpendicular to the flight line, the Doppler centroid $f_{\mathrm{DC}}$ is ideally zero. However, if the antenna is off-set in angle (squinted), which might be due to desired or undesired yaw or if a satellite SAR orbiting a rotating Earth is considered, then $f_{\mathrm{DC}}$ will be different from zero.

In principle, it is possible to calculate the Doppler centroid from orbit and attitude data, but measurement uncertainties on these parameters (primarily attitude) will limit the accuracy. This estimation error will eventually degrade the performance of the processing, especially with respect to signal-to-noise ratio, sidelobe and ambiguity levels. Alternatively, the Doppler centroid can be estimated from the received complex echo data. This has previously been done by frequency analysis of the data in the along-track dimension [3-6].

However, since the frequency resolution and the accuracy of the periodogram (the estimated spectrum) improve with the length and the number of FFTs (FFT is fast Fourier transform), respectively, the frequency domain techniques lead to a significant amount of computations and a storage requirement that might be problematic in relation to a real time processing implementation.

These problems can be circumvented by using time-domain techniques to estimate the Doppler centroid. 


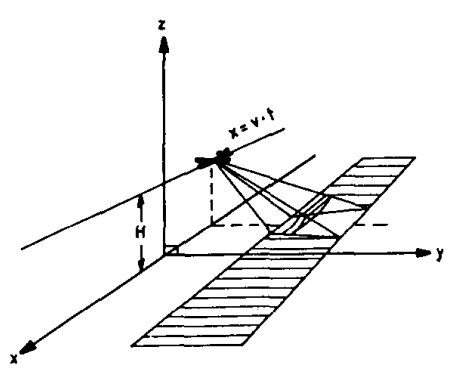

Fig. 1. Geometry of strip map SAR.

The basic idea of a time-domain technique is to exploit the fact that the autocorrelation function of a signal is related to the power spectrum by a Fourier transformation. Hence, shifting the signal in the frequency domain imposes a linear phase-shift on the correlation coefficients, which gives a direct measurement of the size of the frequency shift.

Furthermore, it is possible by exploiting the statistical properties of SAR images, to estimate the correlation coefficients on the basis of the signs of the data values, and this reduces the sensitivity of the cstimator to bright targets. Previously, very complex algorithms were required to obtain an estimator that was robust to significant scene variations, [6].

In Section II a SAR system model is briefly outlined. Then Section III reviews previously used frequency-domain techniques. In Section IV the proposed time-domain techniques are outlined and Section V concentrates on the nonlinear "Sign-Doppler Estimator." In Section VI an evaluation based on an existing SEASAT processor is reported. Finally, the presented work is summarized and concluded in Section VII.

\section{SAR SYSTEM MODEL}

There are basically two problems to be treated in this section. One is to relate the scene reflectivity and the complex output image, that is, the nondetected image. Secondly, a model for the radar scene is required. The first problem has been treated in [7-9] whereas the latter problem is analyzed in [2], and summarized in [10].

To limit the discussion, the azimuth and range channels are assumed independent, which in essence reduces the analysis to one dimension. This simplification has no impact on the principles of the methods presented. The full analysis is given in [2].

The phase history of a target positioned at $\mathbf{R}_{T}(t)$ is given by the distance to the radar platform positioned at $\mathbf{R}_{P}(r)$. The phase shift due to the signal time delay is

$$
\Phi(t)=-\frac{4 \pi}{\lambda}\left|\mathbf{R}_{T}(t)-\mathbf{R}_{P}(t)\right|
$$

Since only the relative positions are of importance we define

$$
\mathbf{R}_{r}(t)=\mathbf{R}_{T}(t)-\mathbf{R}_{P}(t)
$$

Due to the antenna radiation beam, the phase history is only observed in a short time interval centered at $t_{0}$, the moment where the target is in the antenna boresight direction. Hence we can Taylor expand $\mathbf{R}_{r}(t)$ to give

$$
\mathbf{R}_{r}(t) \simeq \mathbf{R}_{r}\left(t_{0}\right)+\left(t-t_{0}\right) \mathbf{V}_{r}\left(t_{0}\right)+\frac{1}{2}\left(t-t_{0}\right)^{2} \mathbf{A}_{r}\left(t_{0}\right) .
$$

We can find the instantaneous Doppler-shift from

$$
f_{\mathrm{Di}}(t)=\frac{1}{2 \pi} \frac{d \Phi(t)}{d t}
$$

Retaining only terms to first order in $t$ (which can be shown to be sufficient, [5]) we find

$$
\begin{aligned}
f_{\mathrm{Di}}(t) & =f_{\mathrm{DC}}\left(t_{0}\right)+\left(t-t_{0}\right) f_{\mathrm{DR}}\left(t_{0}\right) \\
f_{\mathrm{DC}}\left(t_{0}\right) & =-\frac{2 \mathbf{R}_{r}\left(t_{0}\right) \cdot \mathbf{V}_{r}\left(t_{0}\right)}{\lambda\left|\mathbf{R}_{r}\left(t_{0}\right)\right|} \\
f_{\mathrm{DR}}\left(t_{0}\right) & =-\frac{2\left(\mathbf{V}_{r}\left(t_{0}\right) \cdot \mathbf{V}_{r}\left(t_{0}\right)+\mathbf{R}_{r}\left(t_{0}\right) \cdot \mathbf{A}_{r}\left(t_{0}\right)\right)}{\lambda\left|\mathbf{R}_{r}\left(t_{0}\right)\right|} .
\end{aligned}
$$

The azimuth Doppler history is thus a linear chirp centered at $f_{\mathrm{DC}}$ having a Doppler rate $f_{\mathrm{DR}}$ and the amplitude function is given by the antenna pattern. Note that both $f_{\mathrm{DC}}$ and $f_{\mathrm{DR}}$ are functions of time. For airborne systems and for spacecrafts in circular orbits $f_{\mathrm{DR}}$ (at a given range) is fairly constant and if ephemeris data do not give the required accuracy then $f_{\mathrm{DR}}$ can be estimated by using autofocus techniques $[4,5]$. By ignoring that $f_{\mathrm{DR}}$ actually varies with range we can model the SAR system as shown in Fig. 2(a) where $\sigma_{c}(x, r)$ is the complex radar cross section $\left(\left|\sigma_{c}(x, r)\right|^{2}=\sigma(x, r)\right)$ in azimuth and slant range coordinates, $f_{x}$ and $f_{r}$ are frequencies in the azimuth and range dimensions, $H_{e}$ represents the encoding of the scene, $n(x, r)$ indicates thermal noise, $H_{c 1}$ is the range compression, $H_{c 2}$ is the azimuth compression, and $f_{\mathrm{DP}}$ is the Doppler-shift of the processor. Our simpler one-dimensional model is shown in Figure 2(b).

The second problem was how the input scene $\sigma_{c}(\mathbf{r})$ should be modeled. Except when corner reflectors are present, the radar resolution cell comprises a number of scatterers. These will add with random phase, and the resultant of this random walk process is a circular symmetric Gaussian process, [2, 11]. Furthermore, since an SAR usually cannot resolve the individual scatter contributions, then a nonstationary white noise process with circular symmetric Gaussian statistics will model the scene well. The correlation properties of the complex backscatter coefficient are related to the average backscatter coefficient $\sigma^{0}(\mathbf{r})$ by

$$
R_{\sigma_{c}}(\mathbf{r}+\Delta \mathbf{r}, \mathbf{r})=E\left\{\sigma_{c}(\mathbf{r}+\Delta \mathbf{r}, \mathbf{r}) \sigma_{c}^{*}(\mathbf{r})\right\}=\sigma^{0}(\mathbf{r}) \delta(\Delta \mathbf{r})
$$




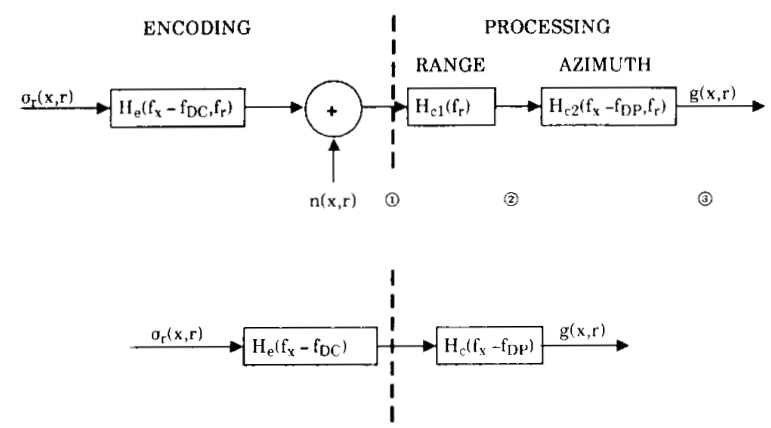

Fig. 2. (a) SAR encoding and processing. (1), (2), and (3) indicate possible points for observing Doppler centroid.

(b) One-dimensional model for SAR encoding and processing.

where $E\{\}$ denote ensemble average. In the next section we see that large variations in $\sigma^{0}$ increase the estimation error on linear Doppler estimators.

As indicated already in Fig. 2(a) there are several possible points where the Doppler centroid can be observed and estimated. Generally the raw SAR video (point (1)) or the range and azimuth compressed data (point (3) should be used. The main advantages of using the raw data are that the estimate will be available before the azimuth compression takes place and the data spectrum will not depend on the processor setting $f_{\mathrm{DP}}$. However, analyzing the raw dispersed data gives substantial errors when bright target responses are only partially covered by the windows used to estimate the Doppler centroid. This means that very long estimation windows (compared with the aperture length) must be used to achieve good precision. This problem is circumvented by estimation based on the compressed data, which, however, require that $f_{\mathrm{DP}}$ is taken into consideration.

\section{FREQUENCY-DOMAIN ESTIMATION}

Since the input to the SAR processor, $\sigma_{c}(\mathbf{r})$, is a "white" random process, then the power spectrum of the complex data will at any stage represent the power transfer function of the signal encoding.

Hence, we can calculate the Fourier Transform of an azimuth line (that is at constant range) of the raw data and the symmetry axis of the spectrum will provide an estimate of the Doppler centroid. Since the variance of a single periodogram will be very large, the estimated power spectrum for a number of neighbouring azimuth lines is generated and averaged to give a better estimate. Still, the above-mentioned problem of partial coverage means that either must long along-track estimation windows be used or the estimation will be very sensitive to nonhomogeneitics in the scene. To avoid this, the estimation can be based on the processed data, as described in $[4,5$, and 6$]$.

By processing the raw data with different $f_{\mathrm{DP}}$ values, and for each processing calculate the energy
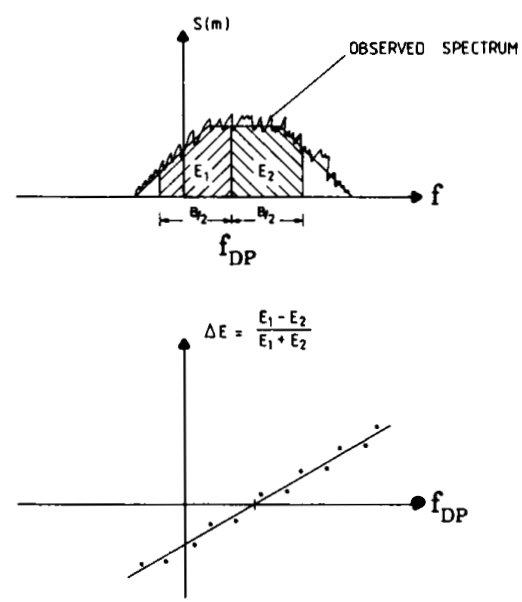

Fig. 3. The $\Delta E$ method for Doppler centroid estimation consists of (a) calculating $\Delta E$ for a number of $f_{\mathrm{DP}}$ values, and (b) determining the $f_{\mathrm{DP}}$ value corresponding to $\Delta E=0$.

difference, $\Delta E$, of two bands placed below and above $f_{\mathrm{DP}}$, respectively, the $\Delta E$ can be mapped as a function of $f_{\mathrm{DP}}$ (see Fig. 3). The estimate for $f_{\mathrm{DC}}$ is the value of $f_{\mathrm{DP}}$ giving $\Delta E=0$. Since $\Delta E$ is a linear function of $f_{\mathrm{DP}}$ in an interval near $\Delta E=0$ then the efficiency of the algorithm can be improved if reasonable Doppler-estimates are available (e.g., from precise ephemeris data or neighbor images). First, the differential coefficient

$$
\left|\frac{\partial \Delta E}{\partial f_{\mathrm{DP}}}\right|_{\Delta E=0}=\epsilon
$$

is found either experimentally or from theory (see [2]). Then the Doppler estimate can be found

$$
\hat{f}_{\mathrm{DC}}=f_{\mathrm{DP}}-\frac{\Delta \hat{E}\left(f_{\mathrm{DP}}\right)}{\epsilon}
$$

By using the complex Gaussian circular symmetric model for $\sigma_{c}(\mathbf{r})$ and equation (8) it is possible (though tedious) to calculate the standard deviation of the estimator $\hat{f}_{\mathrm{DC}}$ under the assumption that the scene is quasi-stationary, that is, $\sigma_{0}(\mathbf{r})$ is slowly varying compared with the system impulse response. This has been done in [2], and one of the most important results is that the variance of the estimator is proportional to the scene contrast, $\mathrm{SC}$, defined as

$$
\mathrm{SC}=\frac{\left\langle\sigma^{0}(\mathbf{r})^{2}\right\rangle}{\left\langle\sigma^{0}(\mathbf{r})\right\rangle^{2}}
$$

where \langle\rangle indicates spatial averaging.

\section{TIME-DOMAIN ESTIMATION}

An alternative way of finding the Doppler centroid is by using the Fourier relation between a signal power spectrum and correlation function. 
If $h_{0}(n)=h_{0}^{\prime}(n T)$ is a stochastic process, its correlation function of two samples separated by $k$ is given by

$$
R_{0}(k)=E\left\{h_{0}(k+m) h_{0}^{*}(m)\right\}
$$

and the power spectrum is given by

$$
\begin{gathered}
S_{0}(f)=F\left\{R_{0}(k)\right\}=\sum_{k=-\infty}^{\infty} R_{0}(k) e^{-j 2 \pi k T f} \\
\Leftrightarrow R_{0}(k)=F^{-1}\left\{S_{0}(f)\right\}=T \int_{-1 / 2 T}^{1 / 2 T} S_{0}(f) e^{j 2 \pi k T f} d f .
\end{gathered}
$$

Now, if we shift the spectrum so that we have

$$
S_{h}(f)=S_{0}\left(f-f_{\mathrm{DC}}\right)
$$

then the correlation function changes

$$
R_{h}(k)=e^{j 2 \pi k T f_{\mathrm{DC}}} R_{0}(k) .
$$

This result is the basis of the time-domain estimation of the Doppler centroid. It is seen that the phase of the correlation function is directly related to the Doppler centroid. Therefore one can by estimation of the correlation function $R_{h}(k)$ arrive at an estimate of the Doppler centroid. The correlation coefficient can easily be estimated as

$$
\hat{R}_{h}(k)=\frac{1}{N} \sum_{i=1}^{N} h(k+m) h^{*}(m)
$$

and the estimator for the Doppler centroid is then

$$
\hat{f}_{\mathrm{D}}=\frac{1}{2 \pi k T} \arg \left\{\hat{R}_{h}(k)\right\}
$$

In conclusion it is possible in a real-time system to keep track of the Doppler centroid by continuously estimating a single correlation coefficient. The algorithm is denoted the correlation Doppler estimator (CDE).

It is worth considering which correlation coefficient $R_{h}(k)$ should be used. Two things are especially important in that respect. One is that the unambiguous Doppler range of $R_{h}(k)$ is

$$
\Delta f_{\text {DC, unamb }}=\frac{1}{k T} \text {. }
$$

Secondly, by doing an in-depth analysis of the standard deviation of the Doppler estimator it is found that when the estimation noise on the argument of $\hat{R}_{h}(k)$, here called $\phi$, is small compared with $1 \mathrm{rad}$ (which it is if the estimator should be useful at all), then one can derive

$$
\operatorname{var}\{\phi\} \leq \mathrm{SC} \frac{1}{N T} \frac{\int S_{0}(f)^{2} d f}{\left|R_{h}(k)\right|^{2}} .
$$

From equation (18) we find

$$
\operatorname{var}\left\{\hat{f}_{\mathrm{DC}}\right\}=\frac{1}{(2 \pi k T)^{2}} \operatorname{var}\{\phi\}
$$

By combining (20) and (21) we have

$$
\sigma\left(\hat{f}_{\mathrm{DC}}\right) \leq \frac{\text { constant }}{\left|k R_{0}(k)\right|} .
$$

Since most SAR systems are only sampled slightly above the Nyquist rate then $R_{0}(k)$ goes rapidly to zero when $|k|$ increases and hence $k=1$ will usually be preferred. In the following we assume $k=1$.

For the same reasons as mentioned above, it can be advantageous to use the processed data and avoid partial coverage of bright targets. In this case the compression filter contributes to the resulting power spectrum. This is described by

$$
S_{h}(f)=S_{e}\left(f-f_{\mathrm{DC}}\right) S_{c}\left(f-f_{\mathrm{DP}}\right)
$$

where $S_{e}(f)$ and $S_{c}(f)$ are the power transfer functions of the encoding and the compression, respectively. This in effect makes the argument of $R_{h}(k)$ a function of both $f_{\mathrm{DC}}$ and $f_{\mathrm{DP}}$, since we have

$$
\begin{aligned}
R_{h}(k) & =T \int_{-1 / 2 T}^{1 / 2 T} S_{e}\left(f-f_{\mathrm{DC}}\right) S_{c}\left(f-f_{\mathrm{DP}}\right) e^{j 2 \pi k T f} d f \\
& =e^{j 2 \pi k T f_{\mathrm{DC}}} T \int_{-1 / 2 T}^{1 / 2 T} S_{e}(f) S_{c}(f-\Delta f) e^{j 2 \pi k T f} d f \\
\Delta f & =f_{\mathrm{DP}}-f_{\mathrm{DC}} .
\end{aligned}
$$

Now, if we assume that $S_{e}(f)$ and $S_{c}(f)$ are symmetrical (which they practically always are by design) then the argument of the last integral is zero for $\Delta f=0$, and for small $\Delta f$ the argument is linear in $\Delta f$. (In practice the linearity is a good approximation over a significant range). So, similar to the frequency-domain technique we can select to either 1) plot $f_{\mathrm{D}}^{\prime}=(2 \pi k T)^{-1} \arg \left\{R_{h}(k)\right\}$ as a function of $f_{\mathrm{DP}}$, and the correct Doppler estimate will then be given by $f_{\mathrm{D}}^{\prime}=f_{\mathrm{DP}}=f_{\mathrm{DC}}$, or 2) exploit the linearity in $\Delta f$ of the integral argument, meaning that

$$
\begin{aligned}
f_{\mathrm{D}}^{\prime} & =\frac{1}{2 \pi k T} \arg \left\{R_{h}(k)\right\} \approx f_{\mathrm{DC}}+\alpha \Delta f \\
\Rightarrow f_{\mathrm{DC}} & \approx \frac{f_{\mathrm{D}}^{\prime}-\alpha f_{\mathrm{DP}}}{1-\alpha} .
\end{aligned}
$$

This constant of proportionality $\alpha$ can be found by using either experimental or theoretical techniques. In practice, theory and experiments give very similar results [2]. It is possible to calculate the variance of the estimator given by (25) and (26) under the assumption that the scene is quasi-stationary, and like the $\Delta E$ method it is found that the variance is proportional to the scene contrast SC given in (11). By using a nonlinear method to estimate the correlation a more "robust" estimator can be designed as we shall see in the following. 


\section{SIGN-DOPPLER ESTIMATOR}

When circular symmetric Gaussian processes are filtered in linear systems, then the output is also circular symmetric Gaussian. Due to the Gaussian property it is, however, possible to derive the correlation properties of a process by analyzing the sign alone. This means first of all that the majority of calculations involved in the estimation are extremely simple (that is, sign comparisons) and therefore the algorithm is well suited to real-time implementations. Secondly, the estimator does not give more weight to bright targets, which makes it less sensitive to strongly varying scenes.

The basic idea is to use the "arcsine law" of Gaussian processes. The arcsine law is derived in [12]. The fundamental result is that when $x(t)$ and $y(t)$ are real Gaussian processes with zero mean and standard deviation $\sigma$, then the normalized correlation coefficient is

$$
\rho_{x y}(t)=\frac{E\{x(t+\tau) y(t)\}}{\sqrt{E\left\{x(t+\tau)^{2}\right\} E\left\{y(t)^{2}\right\}}}=\frac{R_{x y}(\tau)}{\sigma^{2}} .
$$

If we define the sign functions of $x$ and $y$ as

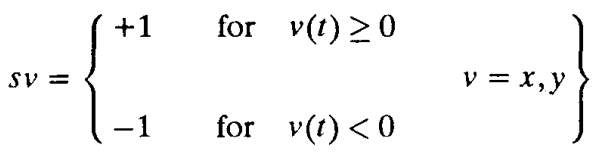

then the arcsine law gives the sign correlation as

$$
\begin{aligned}
R_{s x s y}(\tau) & =\frac{2}{\pi} \arcsin \left\{\rho_{x y}(\tau)\right\} \\
\rho_{x y}(\tau) & =\sin \left\{\frac{\pi}{2} R_{s x s y}(\tau)\right\} .
\end{aligned}
$$

By writing a complex number as

$$
h(k)=I(k)+j Q(k)
$$

where $I$ and $Q$ are real, then we derive

$$
\rho_{h}(k)=\frac{1}{2}\left(\rho_{I I}(k)+\rho_{Q Q}(k)\right)+j \frac{1}{2}\left(\rho_{Q I}(k)-\rho_{Q I}(k)\right) .
$$

Now, since the arguments of $R_{k}(k)$ and $\rho_{h}(k)$ are equal, then the Doppler centroid estimate can be derived in the same way as discussed in Section IV. The complete sign-Doppler estimation algorithm is outlined in Fig. 4. It is important to note that the calculation of $R_{s x, s y}(k)$ (step 2, Fig. 4) is in essence a matter of counting how often two numbers, shifted $k$ samples in azimuth, have the same sign. This is a simple logical operation, and hence well suited for real-time implementations.

There is one problem in relation to the nonlinear estimation algorithm, which is that a general expression for the standard deviation of the estimator has not been found. However, in specific cases, such as the SEASAT example presented in Section VI, approximate figures can be derived. It is important to

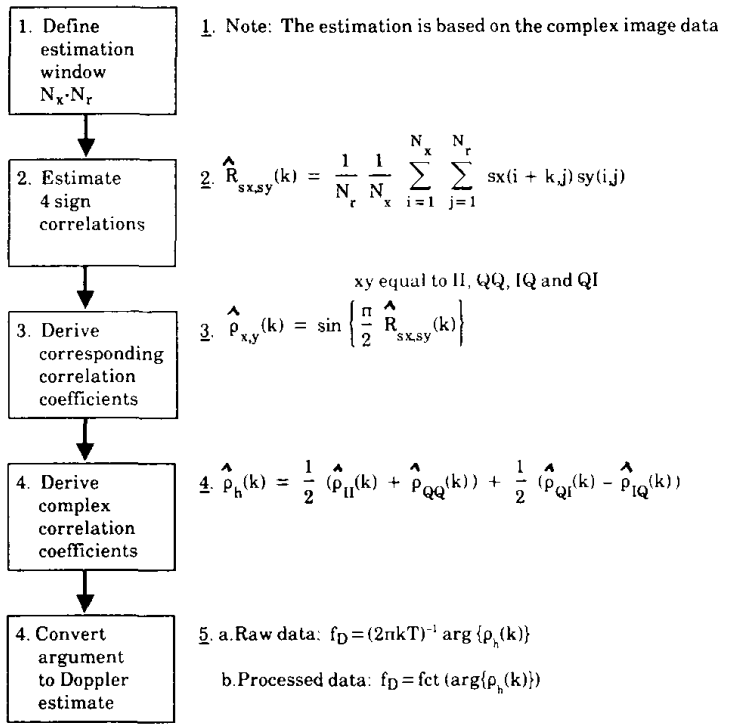

Fig. 4. Flowchart for sign-Doppler estimation algorithm.

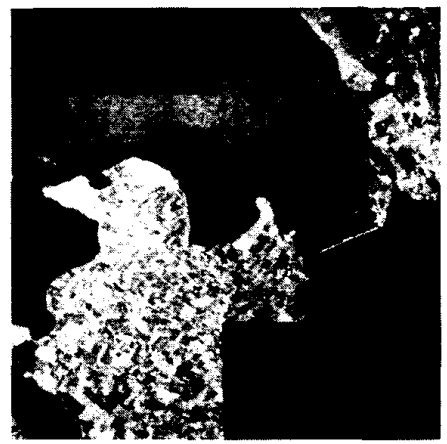

Fig. 5. SEASAT SAR image covering parts of islands Sjaelland and Falster.

note that the scene contrast $\mathrm{SC}$ is not appearing in these expressions.

\section{EVALUATION OF DOPPLER TRACKERS}

The Doppler estimation algorithms presented in the previous sections have been tested on data from the SEASAT satellite SAR. The data cover part of the Danish island Sjaelland, inland waters and the island Falster, Fig. 5. Obviously it would be desirable to perform a more extended test including a variety of scene types. Lack of data has, however, prevented that so far.

The estimators were incorporated in the SEASAT processor of the Electromagnetics Institute [13]. This is a one-look processor that processes data in a slant range/azimuth format to a resolution of 7.5 $m$ in both dimensions. The size of the estimation windows were 2048 by 64 samples in azimuth and range, respectively. The scene can be described as relatively nonhomogeneous, though not extreme, with agricultural land areas, towns, and both windy and calm waters. 
TABLE I

Standard Deviation Of Doppler Estimators

\begin{tabular}{ccccc}
\hline & \multicolumn{2}{c}{ Image 1 } & \multicolumn{2}{c}{ Image 2 } \\
\cline { 2 - 5 } & Exp. & Theory & Exp. & Theory \\
\hline$\Delta E$ & 8.1 & 4.9 & 10.0 & 5.6 \\
$\mathrm{CDE}$ & 8.2 & 3.9 & 10.0 & 4.5 \\
$\mathrm{SDE}$ & 5.9 & 4.1 & 6.6 & 4.1 \\
\hline
\end{tabular}

The evaluation of the accuracy of the estimators follows the approach of [5]. In short, the principle is to analyze a number of windows which are lying next to each other in range. By theory, the Doppler centroid will be very close to a linear function of range. Therefore, by fitting the estimated Doppler centroids to a linear function of range and calculating the rms deviation of the observations relative to the fit, an indication of the estimator standard deviation is achieved. This evaluation procedure was used on two image subsections, each containing 15 estimation windows consecutive in range. The results of the experimental evaluation are shown in Table I together with theoretically predicted values. It is interesting to note the following. 1) The performance of the $\Delta E$ and the CDE estimators which are both linear are very similar, and 2) for this given nonhomogeneous scene, the SDE performs significantly better than both linear algorithms.

It is also obvious that the theoretical predictions (taken from [2]) are too low. This is explained by two facts. One is that the analysis did not include thermal noise, which is especially relevant when doing the linearizing approximations, (10) and (26), since $\epsilon$ and $\alpha$ will be functions of SNR. Secondly, to calculate theoretically the estimator standard deviations quasi-stationarity of the scene was assumed, which is of course a rough approximation when point targets are present also.

It is also interesting to compare the computational load of the estimation algorithms. An estimation window of 64 samples (range) by 2048 samples (azimuth) gives the following figures.

$\Delta E$ method: One complex FFT of length $N=$ 2048 requires $N / 2 \log _{2} N$ butterflies (1 butterfly $=4$ real multiplications and 6 real adds (or subtracts)). Hence, 64 FFTs of length 2048 gives $2.88 \cdot 10^{6}$ multiplications and $4.33 \cdot 10^{6}$ adds. Detection of the 64 FFT lines requires $0.52 \cdot 10^{6}$ multiplications and $0.26 \cdot 10^{6}$ adds. Finally, averaging the 64 lines requires $0.26 \cdot 10^{6}$ adds. The calculation of $\Delta E$ and $f_{\mathrm{DC}}$ (see (10)) does not give a noteworthy contribution. The total computational load per block is therefore $4.85 \cdot 10^{6}$ real additions and $3.40 \cdot 10^{6}$ multiplications.
CDE method: The estimator given by (17) involves $0.26 \cdot 10^{6}$ complex multiplications and the same number of complex additions. Compared with this the derivation of the argument (see (18)) is negligible. In total the computational load per block is $1.05 \cdot 10^{6}$ real multiplications and $1.05 \cdot 10^{6}$ real additions.

SDE method: From Fig. 4 it is easily seen that the $\mathrm{SDE}$ algorithm requires $1.05 \cdot 10^{6}$ sign comparisons and $1.05 \cdot 10^{6} 1$ bit adds (which can be implemented as a counter). Again the remaining derivations of the argument (steps 2, 4, and 5 in Fig. 4) can be neglected. In total one block requires $1.05 \cdot 10^{6}$ sign comparisons and $1.05 \cdot 10^{6} 1$ bit adds. Since this method only relies on the sign bit it is not only computationally efficient but also very efficient with respect to the memory requirements.

\section{CONCLUSIONS}

In the paper three Doppler centroid estimators were discussed. The $\Delta E$ method, originally proposed by JPL, is a frequency-domain technique. The CDE and the SDE, both operating in the time domain, have not previously been proposed for SAR Doppler trackers.

The time-domain algorithms are extremely efficient with respect to requirements on calculations and memory, and hence they are well suited to real-time systems where the Doppler estimation is based on raw SAR data.

For off-line processors where the Doppler estimation is performed on processed data, which removes the problem of partial coverage of bright targets, then the $\Delta E$ estimator and the CDE algorithm give similar performance. However, when it comes to nonhomogeneous scenes, it is found that the nonlinear SDE algorithm, which estimates the Doppler-shift on the basis of data signs alone, gives superior performance. Previously very complex algorithms were required to achieve "optimal" performance in nonhomogeneous scenes [6].

At present a real-time version of the SDE is being implemented in an airborne high-resolution $C$-band SAR, which is currently being built in Denmark.

\section{ACKNOWLEDGMENTS}

The author wishes to thank Jørgen Dall, The Electromagnetics Institute, Technical University of Denmark, for integrating the various Doppler trackers in the Danish SEASAT processor. Also, the comments and suggestions of Dr. John Curlander of the Jet Propulsion Laboratory, are appreciated. 


\section{REFERENCES}

[1] Ulaby, F. T., Moore, R. K., and Fung, A. K. (1983) Microwave Remote Sensing, Active and Passive, Vol. II. Reading, Mass., Addison Wesley, 1983.

[2] Madsen, S. N. (1986) Speckle theory, modelling, analysis, and applications related to synthetic aperture radar data.

Ph.D. thesis (report LD62), Electromagnetics Institute, Lyngby, Denmark, Nov. 1986.

[3] Cumming, I. G., and Bennett, F. R. (1979)

Digital processing of SEASAT SAR data.

In Proceedings of the International Conference on Acoustics, Speech and Signal Processing, Washington, D.C., Apr. 1979, pp. 710-718.

[4] Curlander, J. C., Wu, C., and Pang, A. (1982) Automatic preprocessing of spaceborne SAR data. In Proceedings of the 1982 International Geascience and Remote Sensing Symposium, Munich, W. Germany, June 1982, pp. 3.1-3.6.

[5] Li, F. K., Held, D. N., Curlander, J. C., and Wu, C. (1985) Doppler parameter estimation for spaceborne synthetic-aperture radars. IEEE Transactions on Geascience and Remote Sensing, GE-23, 1 (Jan. 1985), 47-56.

[6] Jin, M. Y. (1986)

Optimal Doppler centroid estimation for SAR data from a quasi-homogeneous source.

IEEE Transactions on Geoscience and Remote Sensing, GE-24, 6 (Nov. 1986), 1022-1025.

[7] Van de Lindt, W. J. (1977)

Digital technique for generating synthetic aperture radar images.

IBM Journal of Research and Development (Sept. 1977), $415-432$.
[8] Wu, C., Liu, K. Y., and Jin, M. Y. (1982)

Modelling and a correlation algorithm for spaceborne SAR signals.

IEEE Transactions on Aerospace and Electronic Systems, AES-18, 5 (Sept. 1982), 563-575.

[9] Ausherman, D. A., Kozma, A., Walker, J. L., Jones, H. M., and Poggio, A. (1984)

Developments in radar imaging.

IEEE Transactions on Aeraspace and Electronic Systems, AES-20, 4 (July 1984), 363-398.

[10] Madsen, S. N. (1987)

The average power spectrum of homogeneous and non-homogeneous radar images.

IEEE Transactions on Aeraspace and Electronic Systems, AES-23, 5 (Sept. 1987), 583-588.

[11] Goodman, J. W. (1975)

Statistical properties of laser speckle patterns.

In J. C. Dainty (Ed.), Laser Speckle and Related

Phenomena.

New York: Springer, 1975, pp. 9-75.

[12] Papoulis, A. (1965)

Probability, Random Variables and Stochastic Processes. Ncw York: McGraw-Hill, 1965, pp. 197-198 and 483-485.

[13] Dall, J. (1984)

Digital SEASAT processor.

M.Sc. thesis (report E250) (in Danish), Electromagnetics Institute, Lyngby, Denmark, 1984.

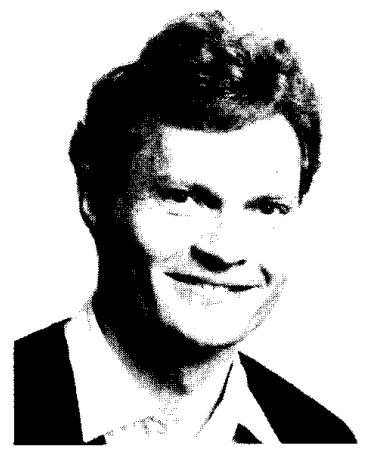

IEEE TRANSACTIONS ON AEROSPACE AND ELECTRONIC SYSTEMS aperture radar images. airborne SAR.
Søren Nørvang Madsen received the M.Sc. degree in electrical engineering and electrophysics from the Technical University of Denmark, Lyngby, Denmark, in 1982 with a thesis on a computer controlled nuclear magnetic resonance spectrometer. In 1987 he received the Ph.D. degree in electrical engineering from the Technical University of Denmark with a thesis on properties of synthetic

He has been with the Electromagnetics Institute, Technical University of Denmark, since 1982. He has been working on research within the field of basic statistics of SAR images, optimal post filtering, and SAR preprocessor developments. He has participated in several field experiments using radar and radiometers for remote sensing of sea ice and oil pollution. Since 1984 he has been an Associate Professor at the Electromagnetics Institute, working primarily with digital signal processing and radar theory. He has also been involved in studies of imaging radars and altimeters for mapping planets of the solar system. $\mathrm{He}$ is currently Project Manager on a project concerned with high resolution 